\title{
Directional and angle-resolved optical scattering of high-performance translucent polymer sheets for energy-efficient lighting and skylights
}

\author{
Jacob C. Jonsson, Geoffrey B. Smith, Christine Deller, and Arne Roos
}

\begin{abstract}
Transparent refractive-index matched micro (TRLMM) particles have proved to be an excellent scattering component for use in translucent sheets. Measurements of hemispheric transmittance and reflectance versus angle of incidence, as well as angle-resolved studies of such translucent sheets, have been carried out to complement earlier published hemispheric reflectance and transmittance spectral measurements carried out at normal angle of incidence. Hemispheric values relative to angle of incidence are of interest for daylighting applications and building simulations, and angle-resolved measurements are vital for verifying that our modeling tools are reliable. Ray-tracing simulations based on Mie scattering for the individual TRIMM particles and angle-resolved measurements are in good agreement, indicating that the simulation method used is practical for the, design of new scattering profiles by varying particle concentration or refractive index. (c) 2005 Optical Society of America

OCIS codes: $\quad 160.4760,160.5470,290.5850,120.6200,290.4020,290.4210$
\end{abstract}

\section{Introduction}

Increased energy costs in combination with limited power capacity and the obligation to honor the Kyoto Protocol has led to increased interest in energyefficient building technologies. An important contribution to a building's annual energy balance is provided by the Sun. Solar irradiation can contribute to heating in the winter but unfortunately also to overheating in the summer. The use of modern solar control glazing with low-E coatings can considerably reduce energy consumption by allowing the solar radiation to enter through windows in the winter or by reflecting most of it back to the outside in the summer. Control of glare and daylighting inside a building are also important issues. It is well known that natural daylight is important for people who spend their time indoors. It is difficult problem to provide natural daylight without creating problems with

J. C. Jonsson (jacob.jonsson@angstrom.uu.se) and A. Roos are with the Department of Engineering Sciences, Uppsala University, P.O. Box 534, SE-751 21 Uppsala, Sweden. G. B. Smith and C. Deller are with the Department of Applied Physics, University of Technology, P.O. Box 123, Broadway, Sydney, New South Wales 2007, Australia.

Received 10 March 2004; revised manuscript received 25 November 2004; accepted 9 December 2004.

$0003-6935 / 05 / 140001-09 \$ 15.00 / 0$

(1) 2005 Optical Society of America glare at the same time. Therefore daylight should be provided by diffuse light, and direct radiation should be avoided. Diffuse light is more relaxing and is also more evenly distributed to different parts of the interior. Diffusing glass and plastic sheets have been available for a long time, but a general problem is enhanced backscattering, which seriously reduces the transmittance of such devices.

This paper is a continuation of our previous study of transparent refractive-index matched micro (TRIMM) particles as the scattering element in translucent sheets for use in lighting applications ${ }^{1}$ and demonstrates and validates a ray-tracing method for simulation of the scattering profile at normal angles of incidence. The initial study was limited to normal-incidence spectral data, and in this paper we examine the actual scattering profile and the effects of varying the angle of incidence for both hemispheric and directional transmittance. A number of applications in lighting technology follow from the ability of these sheets and polymers to scatter almost exclusively in the forward direction. Translucent sheets based on nonabsorbing polymer microparticles randomly dispersed in a transparent polymer have several advantages over traditional pigmented translucent sheets. Low backscattering and negligible absorption are the main advantages achieved by replacing traditional white pigments with TRIMM particles. Not only do these properties yield high 
(a)

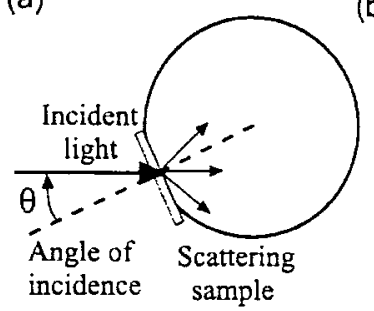

(b)

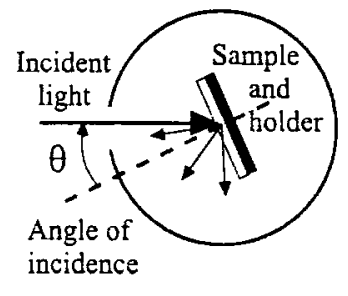

Fig. 1. Top-view schematic of the integrating spheres used for hemispheric measurements of scattering samples at oblique angles of incidence. (a) In the transmittance sphere, both sample and sphere rotate about an axis through the entrance port. (b) For the reflectance sphere, the sample (lighter shading) and the sample holder (darker shading) rotate, but the sphere is fixed. Both figures show the angle of incidence, $\theta$, which is measured with respect to the surface normal.

transmittance but they also allow for easy tailoring of the output-light distribution without adversely affecting the number of lumens transmitted.

The scattering profile in general depends on sheet thickness, particle size, particle concentration, and particle refractive index. In this case particle size is of much less importance than for traditional scatterers, and in large enough particles (greater than approximately $6 \mu \mathrm{m}$ ) it can be neglected. It would be preferable to have a closed analytical expression for the scattering profiles that contain the material parameters of interest but, because of multiple scattering, finding such an expression is not an easy task, if it is possible at all. Instead it is necessary to resort to use of ray-tracing simulation programs for determination of scattering profiles of materials still in the design phase. This approach is clearly preferable to actually producing several components with a wide range of concentrations for experimental characterization.

The TRIMM particle sheets did not show any absorption at normal angles of incidence, but it is of interest to verify that such would also be true for oblique angles of incidence. Loss of transmittance owing to increased specular reflectance from the smooth sample surfaces is expected. Side loss, i.e., light escaping from a sample's sides, can also be expected to increase with increasing angle of incidence.

\section{Theory}

\section{A. Integrating Spheres}

Single-beam integrating spheres, such as those shown in Fig. 1, do not give correct reflectance and transmittance values by simply dividing the sample signal by the reference signal. Corrections used in this paper were derived and are thoroughly described elsewhere, ${ }^{2.3}$ but a short summary is appropriate.

The measured hemispheric transmittance $T_{m}$ (sample-to-reference signal ratio) through a single port tends to be too high for several reasons. The most obvious is that the sample itself reflects light that would otherwise escape from the sphere through the entrance port, which does not happen during the ref-

erence measurement. Corrections are also made for the fact that the diffuse part of the transmitted light is directly scattered by the sample. This is not the case for the specular part and the reference measurement, for which the first sphere wall reflection is needed to diffuse the light such that it enters the detector's field of view. Another correction factor is included to compensate for any non-Lambertian scattering property of the sphere wall. Although it is in general true that a sphere wall is not a perfect Lambertian scatterer, this effect is small and $x$ in Eq. (1) below can be assumed to be equal to 1 . The final expression for the measured transmittance can then be expressed as

$T_{m}=\frac{S_{s}}{S_{r}}=\frac{\left[1-R_{w}\left(1-f_{s}\right)\right]\left\{T_{d}+T_{s}\left[R_{w}\left(1-x f_{s}\right)+x f_{s} R_{s}\right]\right\}}{\left[1-R_{w}\left(1-f_{s}\right)-f_{s} R_{s}\right] R_{w}\left(1-x f_{s}\right)}$

where $S_{s}$ is the signal recorded during the sample measurement, $S_{r}$ is the signal recorded from the reference measurement, $R_{w}$ is the reflectance of the sphere wall, $f_{s}$ is the fraction of the sphere area that is occupied by the sample port, $R_{s}$ is the sample hemispheric reflectance, $x$ is the deviation from Lambertian behavior, and finally $T_{d}$ and $T_{s}$ are the correct diffuse and specular transmittance values, respectively.

By designating the hemispherical transmittance $T_{\text {tot }}=T_{s}+T_{d}=C T_{\text {tot }}+(1-C) T_{\text {tot }}$, where $C$ is the fraction of light that is specularly transmitted, one may solve Eq. (1) for $T_{\text {:ot }}$, which yields

$$
\begin{aligned}
& T_{\text {tot }} \\
& =\frac{S_{s}}{S_{r}} \frac{\left[1-R_{w}\left(1-f_{s}\right)-f_{s} R_{s}\right] R_{w}\left(1-x f_{s}\right)}{\left[1-R_{w}\left(1-f_{s}\right)\right]\left\{C+(1-C)\left[R_{w}\left(1-x f_{s}\right)+x f_{s} R_{s}\right]\right\}}
\end{aligned}
$$

The values of $R_{s}$ and $C$ in Eq. (2) must be measured by other means or estimated by use of prior knowledge about the sample, whereas $R_{w}$ and $f_{s}$ are properties of the integrating sphere used.

The reflectance sphere used ${ }^{3}$ for the experiments reported in this paper was designed with a centermounted sample holder that can be rotated about its own axis. This design, as for the single-beam transmittance sphere, also results in an overestimation of the hemispheric reflectance unless proper corrections are applied. Correction is needed for the different treatments required for specular and diffuse reflectance as well as for port area, sample area, and angle of incidence. The published equation ${ }^{3}$ is applicable only for opaque samples, whereas our samples here are transparent and need an extra term. With an approximate correction for the light that is twice transmitted through the sample and reflected from the sample holder, the full expression for the total reflectance, $R_{\text {tot, }}$, becomes 


$$
R_{\mathrm{tot}}=\frac{R_{w}\left(S_{s} / S_{r}\right)+R_{\mathrm{sp}}\left[1-R_{w}\left(1-A_{2} \cos \theta\right)-R_{h}\left(1-f A_{2} \cos \theta\right]\right.}{1+R_{\mathrm{sp}} f A_{2} \cos \theta}-T_{\mathrm{tot}}^{2} R_{h}
$$

where $f$ is the fraction of the area of the sample holder that is not covered by the sample, $A_{2}$ is the ratio of the sample holder's area to the total sphere area, $\theta$ is the angle of incidence, $R_{h}$ is the reflectance of the sample holder, and all other symbols are as in Eq. (1). It is important to take into consideration that both $T_{\text {tot }}$ and $R_{h}$ vary significantly with angle of incidence. The terms $A_{2}, R_{w}$, and $R_{h}$ are sphere parameters that are all sample independent. Equation (3) lacks any correction for side loss, which is recorded in the $S_{s}$ term because light that exits through the edges is contained inside the sphere and is hence detected.

As $T_{\text {tot }}$ and $R_{\text {tot }}$ depend on each other, it is not obvious how best to find the correct values. A suggested strategy is to measure diffuse and total transmittance and reflectance for normal incidence with a double-beam integrating sphere, which is a standard accessory that is available for most commercial spectrophotometers. This gives information about the relation between scattered and diffuse light (the $C$ factor) as well as a value of $R_{\text {tot }}$ that one can use to find $T_{\text {tot }}$ with Eq. (2). Corrected $R_{\text {tot }}$ values can then be found by use of Eq. (3) for each angle of incidence.

\section{B. Goniometer Scatterometer}

Hemispheric transmittance and reflectance are only partly of interest for the performance of a translucent sheet. The scattering angle profile that shows how diffuse the transmitted light becomes is not determined by use of an integrating sphere. Goniometer scatterometers have to be used to measure the angleresolved scattering intensity and to determine the scattering profile.

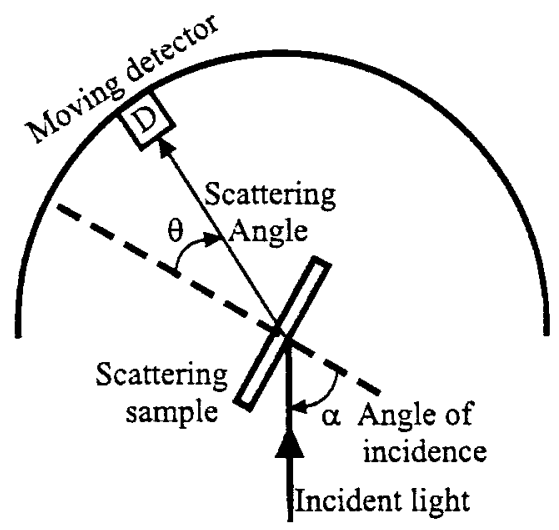

Fig. 2. Schematic of the goniometer setup used for angle-resolved scattering measurements. Angle of incidence $\alpha$ is defined as the angle between the incident light and the sample normal (dashed line). Scattering angle $\theta$ is also defined relative to the surface normal. The sample normal corresponds to $\theta=0^{\circ}$. Positive angles are given by the clockwise direction; negative angles correspond to angles in the counterclockwise direction from the surface normal.
The TRIMM sheets studied here are homogeneous and isotropically scattering; hence it is sufficient to measure only polar angle $\theta$ from zero to $90^{\circ}$ with a fixed angle $\phi$ for the normal angle of incidence. For oblique angles of incidence the scattering profile becomes asymmetric, and a larger part of the hemisphere must be measured. The scattering angle is always calculated from the normal of the sample's surface as defined in Fig. 2.

\section{Ray-Tracing Scattering Model}

Ray-tracing simulations have been used to model the scattering behavior of the TRIMM-particle-doped samples, and the algorithm used to produce a scattering curve is shown in Fig. 3. Mie scattering theory can be used to study a single scattering event, but this does not suffice to describe properly the multiple scattering that occurs in the sheets studied. The simulation was designed such that its results could be compared to such intensity profiles as those recorded with angle-resolved scatterometers, i.e., the intensity for fixed $\phi$ versus scattering angle $\theta$, even though the simulation is carried out in three dimensions and thus allows for rays to emerge at any $\phi$.

\section{Angle-Resolved Light Scattering in Vector Space}

Sorensen and co-workers have demonstrated ${ }^{4-6}$ the benefits of studying Mie scattering with respect to the scattering vector rather than the experimentally more convenient scattering angle. The actual parameter used is the dimensionless quantity $q R$, where $R$ is the particle radius and $q=2 k \sin (\theta / 2)$ is the scattering wave vector for scattering angle $\theta$ and light wave vector $k$. This kind of analysis of the TRIMLM

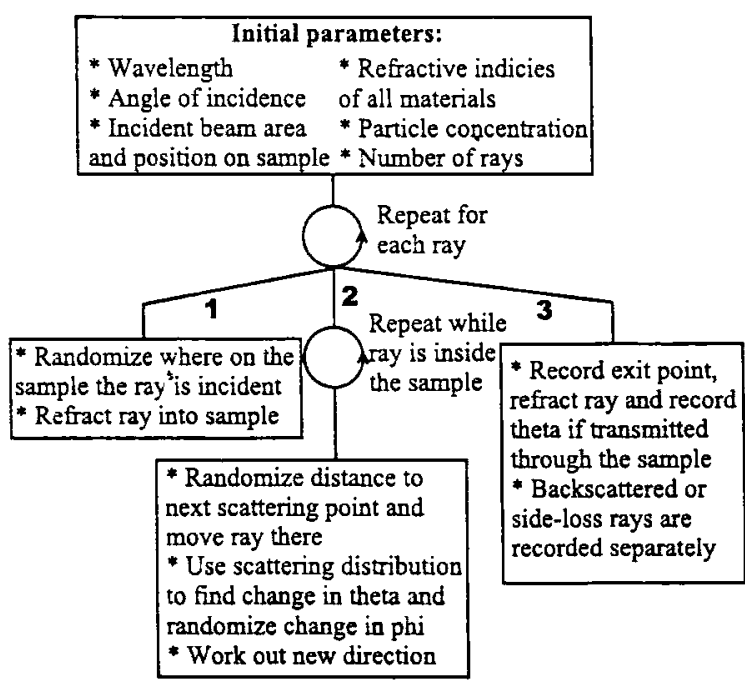

Fig. 3. Flowchart describing the ray-tracing simulation. 
particle sheets is interesting in a wider scope than just this specific sample because it probes the theory of multiple Mie scattering.

The only arguments for using the scattering angle as a variable are that it is intuitive (which is why parts of the data presented in this paper are shown in such a way) and convenient from an experimental point of view, whereas wave vector $q$ combines the scattering angle and the inverse wavelength. The inverse of $q$ is the probe length of the scattering. ${ }^{4} \mathrm{Mul}-$ tiplying $q$ by scattering particle radius $R$, which is also included in Mie theory, yields a dimensionless parameter that incorporates all variables in Mie scattering except the relation between the scatterers and the index of refraction of the medium.

Sorenson and Fischbach have shown that the Mie scattering from a single particle can be divided into three power-law regimes ${ }^{4}$ :

$$
\begin{array}{ll}
I \times(q R)^{0} & q R<1, \\
I \times(q R)^{-2} & 1<q R<\rho, \\
I \times(q R)^{-2} & \rho<q R,
\end{array}
$$

where the phase factor is $\rho=2 k R|m-1|, k$ is the wave vector, $R$ is the particle radius, and $m$ is the ratio between particle and matrix refractive indices.

AQ: B These regimes hold for a single Mie scattering event, but how this translates to multiple scattering has not been thoroughly investigated. Knowledge about this behavior is expected to result in simpler interpretation, even of multiple scattering data. For scattering from particles of different sizes it is plausible to expect a smearing of crossover points, making the transition between one regime to another less sharp. However, in large TRIMM particles the $R$ dependence is weak, but we retain it to allow for some contribution from the minority of particles smaller than $6 \mu \mathrm{m}$.

\section{Experiments and Simulations}

\section{A. Studied Materials}

In this study the number of samples studied was reduced from 12 in the previous paper ${ }^{1}$ to 4 in an attempt to make the results clearer, given the greater complexity of the scattering data. We concentrated our efforts on the poly(methyl methacrylate) (PMMA) $7 \mathrm{~N}$ matrix samples with $1011 \mathrm{~F}$ Plexiglas TRIMM particles as scatterers. The samples have been denoted N73 for a 3\% weight concentration of TRIMM particles and N77 for 7.3\% TRIMM particle concentration. Thicknesses of 1 and $3 \mathrm{~mm}$ were studied for both particle concentrations, and the size of all samples was $5 \mathrm{~cm} \times 5 \mathrm{~cm}$.

The refractive index of the matrix (PMMA 7N) decreased from 1.496 at $400 \mathrm{~nm}$ to 1.488 at $700 \mathrm{~nm}$. The TRIMM particles' refractive index was determined with an Abbe refractometer to be 1.507 at $633 \mathrm{~nm}$,

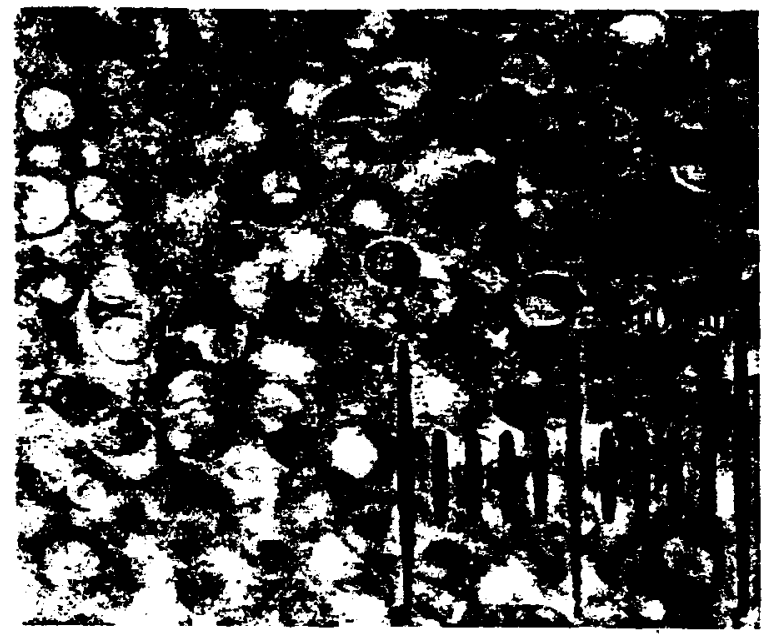

Fig. 4. Micrograph of the N77 sample, showing the smearing of surface particles and an indication of a size distribution among the TRIMM particles. The full scale is $100 \mu \mathrm{m}$ (10 $\mu \mathrm{m}$ per minor tick).

resulting in an $m$ of 1.011 . The dispersion in refractive index of the TRIMM particle material is assumed to correspond to that of the matrix in such a way that $m$ is constant with respect to wavelength.

A micrograph of the near surface of the highconcentration sample (N77) is shown in Fig. 4. The spheres near the surface are expected to be slightly deformed as a result of shear forces applied during injection molding.

\section{B. Hemispheric Peflectance and Transmittance Measurements versus Angle of Incidence}

The transmittance and reflectance for each angle of incidence was measured with $s$ - and $p$-polarized light and calculated according to Eqs. (2) and (3). All values presented are the arithmetic averages of the $s$ and $p$-polarized results.

The entrance port of the transmittance sphere is circular, with a diameter of $42 \mathrm{~mm}$, and the light spot is focused to a diameter of less than $3 \mathrm{~mm}$. Focusing of the incident light causes a slight variation in the angle of incidence of $\pm 1^{\circ}$. The large ratio between port diameter and light-spot diameter means that almost all side loss ${ }^{7}$ will occur through the edge and that there will be none by side-shifted light exiting the front surface. The spot size increases with angle of incidence, and this limits the measurements to an angle of incidence of $70^{\circ}$.

The reflectance measurements were carried out with the same spot size as the transmittance measurements. The sample holder, which is mounted in the center of the reflectance sphere, was covered with black tape on the sample side. The sample was attached with $1 \mathrm{~cm} \times 5 \mathrm{~cm}$ strips of transparent double-sided tape at the upper and lower parts of the holder's surface. This left a small space between the sample and the black tape of the holder. The black tape was characterized for the relevant incidence angles for use in Eq. (3). The double-sided tape behaves 
(a)

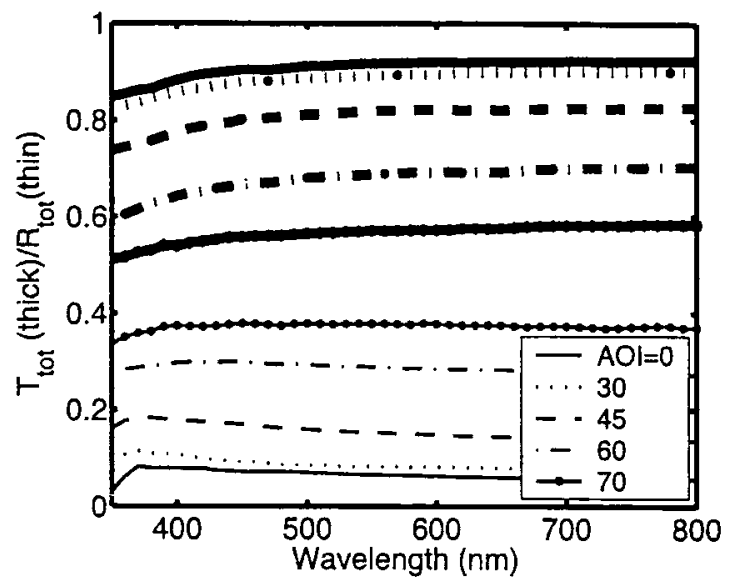

(c)

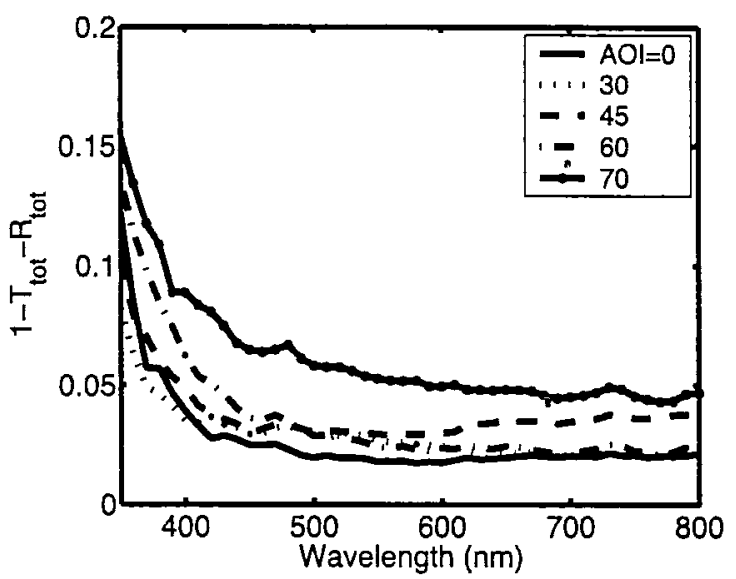

(b)

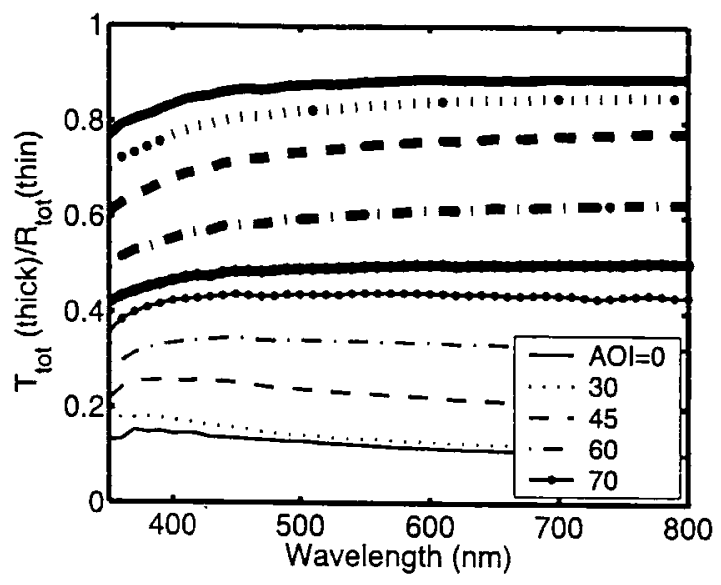

(d)

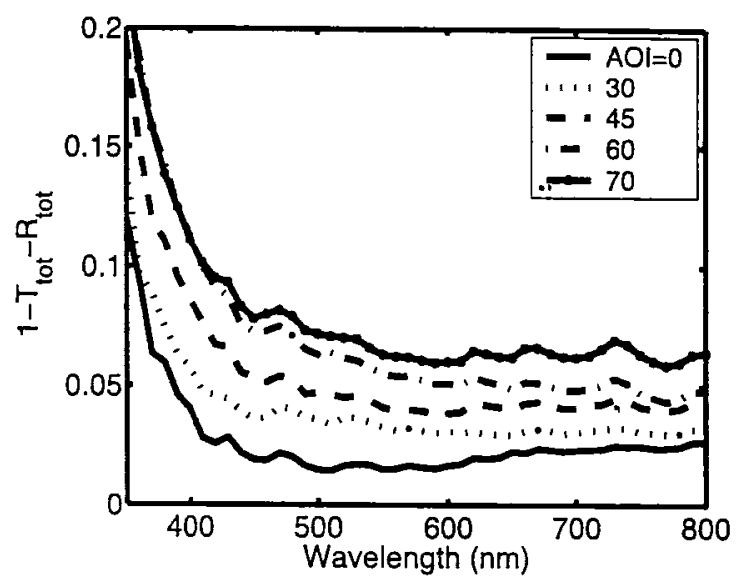

Fig. 5. (a), (b) Hemispheric reflectance (thinner curves) and transmittance (thicker curves) values for several angles of incidence of the N73 samples of thicknesses 1 and $3 \mathrm{~mm}$, respectively. (c), (d) $1-T_{\text {tot }}-R_{\text {tot, }}$ which corresponds to absorption and side loss of the transmittance measurements.

differently from the black tape, mainly in that it sticks to the sample. This complication is ignored because the double-sided tape is assumed to be far enough away from the light spot, an assumption that does not change with increasing angle of incidence, as the light spot's shape is not elongated toward the double-sided tape.

\section{Angle-Resolved Measurements}

All angle-resolved measurements were carried out with a goniometer built at the University of Technology, Sydney. ${ }^{8}$ Because of the instrument's design the measurements were restricted to angles $\theta$ from $-90^{\circ}$ to $70^{\circ}$ while angle $\phi$ was kept fixed. As the samples studied were isotropically scattering, this restriction posed no problem. A fixed $\mathrm{He}-\mathrm{Ne}$ laser was used in combination with a polarizer as a light source for all measurements. Results presented for oblique angles of incidence are arithmetic averages of $s-$ and $p$-polarized measurements.

\section{Ray-Tracing Simulations: Comments on Their Implementation}

We carried out ray-tracing calculations to simulate the $\mathrm{He}-\mathrm{Ne}$ laser goniometer measurements. The most frequent computation task in the program is the scattering event, which is calculated several times for each ray, and optimization of this part was crucial to obtaining the correct results. The core of such an optimization would be to get the random scattering angle with as few run-time calculations as possible from a generated random number, because making full calculations of the complete Mie formalism would be far too time consuming and lead to unreasonable simulation times. The relative intensity for an angle follow from the relative probability that the ray will be scattered in that direction. Knowing this probability, it is possible to create a function that correlates a linear random number to an actual scattering angle. Such a probability function was reduced to a simple lookup vector containing almost $5 \times 10^{6}$ elements to 
(a)

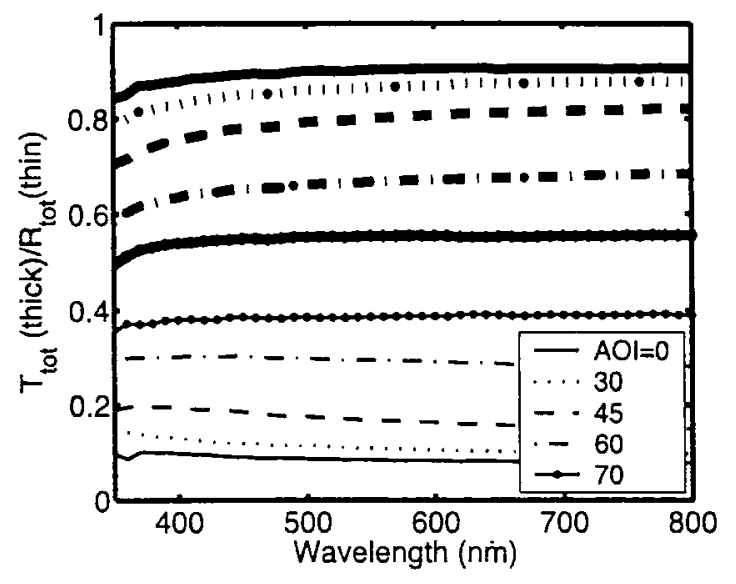

(c)

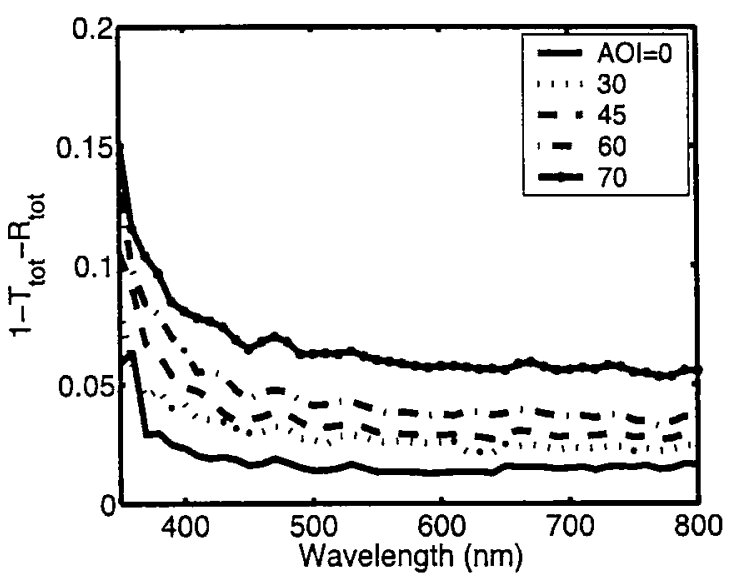

(b)

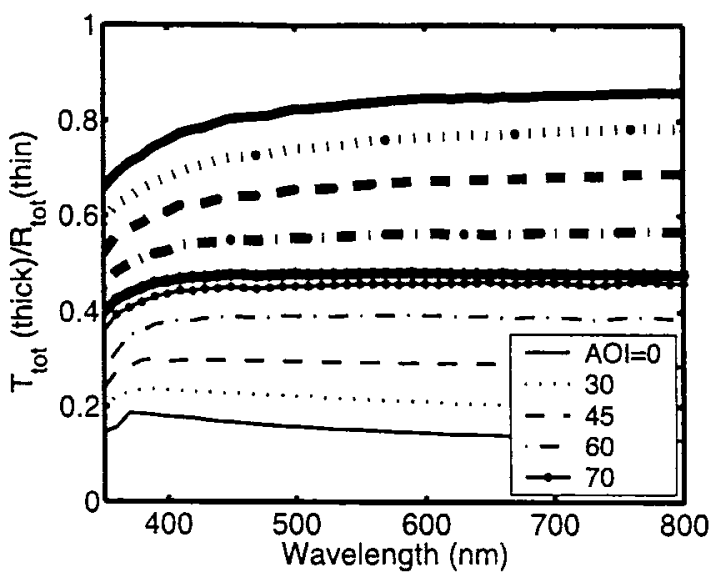

(d)

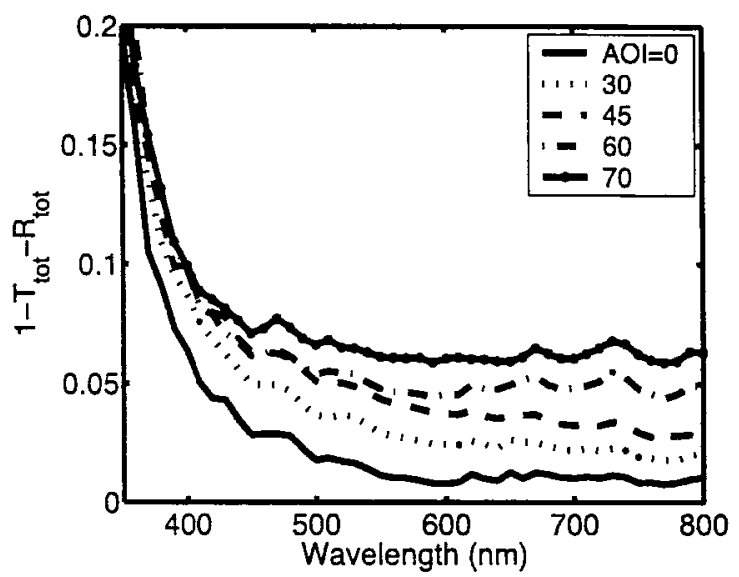

Fig. 6. (a), (b) Hemispheric reflectance (thinner curves) and transmittance (thicker curves) values for several angles of incidence of the N77 samples of thicknesses 1 and $3 \mathrm{~mm}$, respectively. (c), (d) $1-T_{\mathrm{wor}}-R_{\mathrm{ws}}$, which corresponds to absorption and side loss of the transmittance measurements.

maintain a reasonable resolution for high angle scattering. This strategy sped up calculations considerably, requiring only a single scaling multiplication operation of a random number from 0 to 1 to produce a scattering angle. But, as the lookup vectors are rather memory consuming, it is not possible to use a large number of such vectors simultaneously; hence we are limited to a discretized scattering distribution.

Restricting the simulation to a single wavelength simplifies the scattering event not only because the wavelength is constant but also because one does not have to consider dispersion. The particle size distribution for the samples investigated was handled by use of an equal mixture of two discrete sizes, $R$ $=7.5$ and $R=10 \mu \mathrm{m}$. As the ray reaches a particle, the particle's radius is randomized to one of the two discrete sizes, and the angle distribution for that size is used.

As the ray passes through the sample and is scattered, new $\theta$ angles are generated by use of the lookup vector and new $\phi$ angles are randomly generated

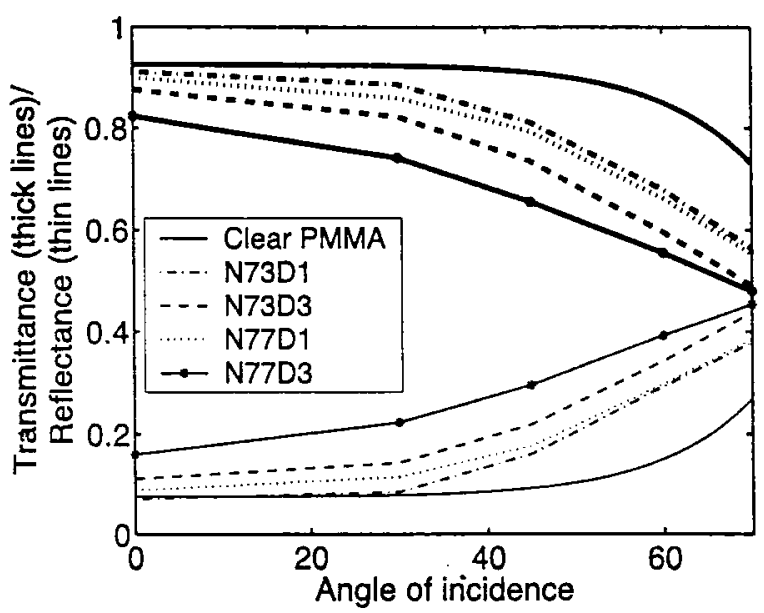

Fig. 7. Hemispheric transmittance and reflectance versus angle of incidence for $500 \mathrm{~nm}$. The solid curves indicate the calculated reflectance for a clear PMMA sample to emphasize the increased effect of scattering with increasing angle of incidence. The large difference between N73D3 and N77D1 indicates that side loss is higher for thicker samples. 
(a)

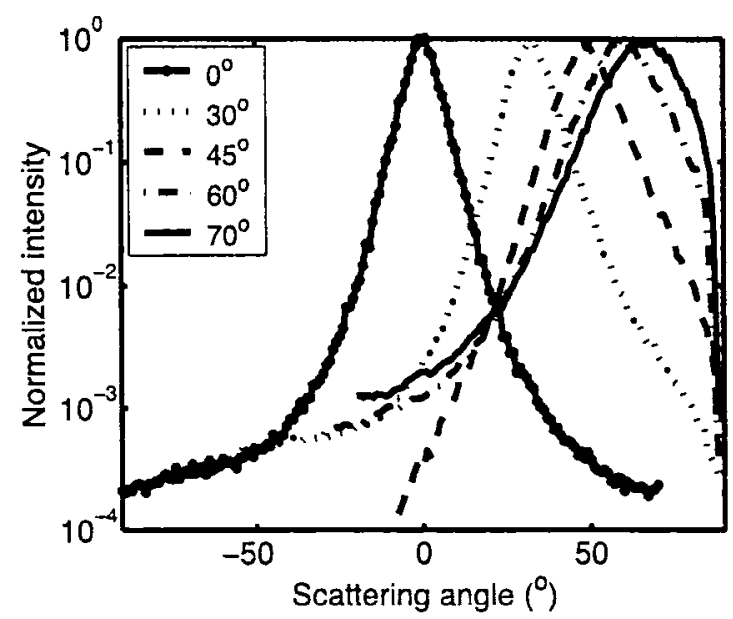

(c)

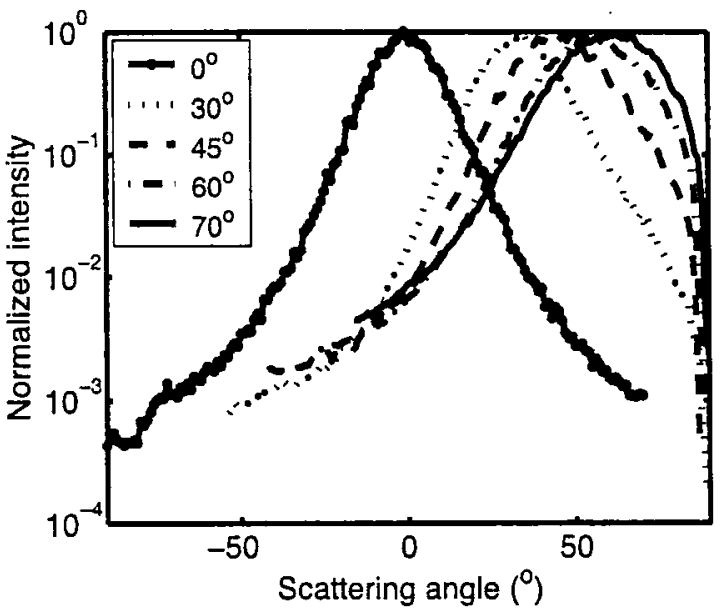

(b)

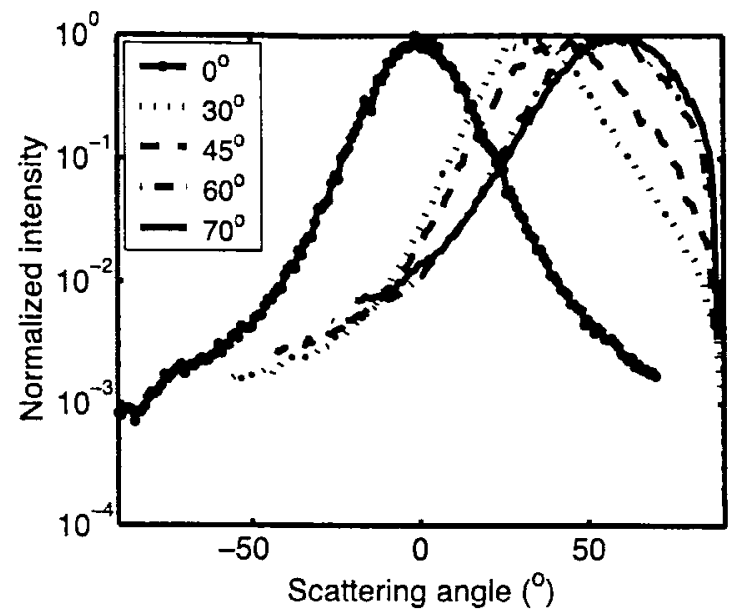

(d)

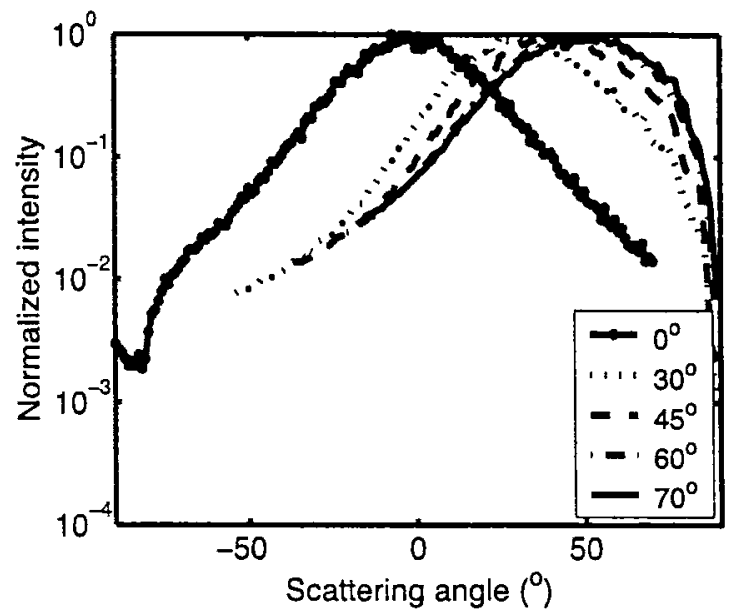

Fig. 8. Experimental results for goniometer measurements of the scattering profiles versus angle of incidence for (a) $\mathrm{N} 73,1 \mathrm{~mm}$; (b) N73, $3 \mathrm{~mm}$; (c) $\mathrm{N} 77,1 \mathrm{~mm}$; and (d) $\mathrm{N} 77,3 \mathrm{~mm}$.

from 0 to $2 \pi$. The new angles $(\Delta \theta$ and $\Delta \phi)$ are added to the ray's orientation by use of spherical geometry according to

$$
\theta_{\text {new }}=\arccos (\cos \theta \sin \Delta \theta+\sin \theta \sin \Delta \theta \cos \Delta \phi),
$$

$$
\phi_{\text {new }}=\arcsin \frac{\sin \Delta \phi \sin \Delta \theta}{\sin \theta_{\text {new }}} .
$$

When the ray is refracted out through the exit surface its $\theta$ angle is recorded by binning with $0.5^{\circ}$ intervals. Rays exiting the side of the sample or backscattered toward the light source are not collected in the forward-scattering bins. The $\phi$ angle value is dropped owing to sample symmetry. As all $\phi$ angles are recorded, the simulated intensity will be higher than the measured intensity because the goniometer uses only a single $\phi$. This increase is proportional to a factor $\sin \theta$, by which the simulation result must be divided to be comparable with the experimental data. The proportionality constant is divided by itself when the intensity is normalized, so it does not need further attention.

\section{Results}

\section{A. Hemisprizic Reflectance and Transmittance} Measurements versus Angle of Incidence

The hemispheric transmittance and reflectance measurements shown in Figs. 5(a), 5(b), 6(a), and 6(b) all follow the expected increase in reflectance, hence decrease in transmittance, as the angle of incidence increases. Figures 5(c), 5(d), 6(c), and 6(d) show the amount of light that is absorbed or undetected owing to side loss. The absorption of PMMA is quite low, but it may increase slightly with increased path length of travel through the samples. However, almost the full 
(a)

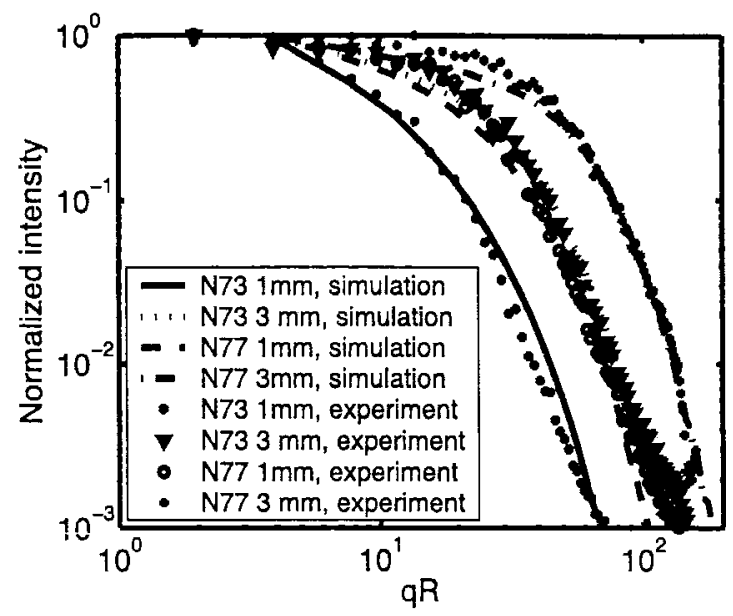

(b)

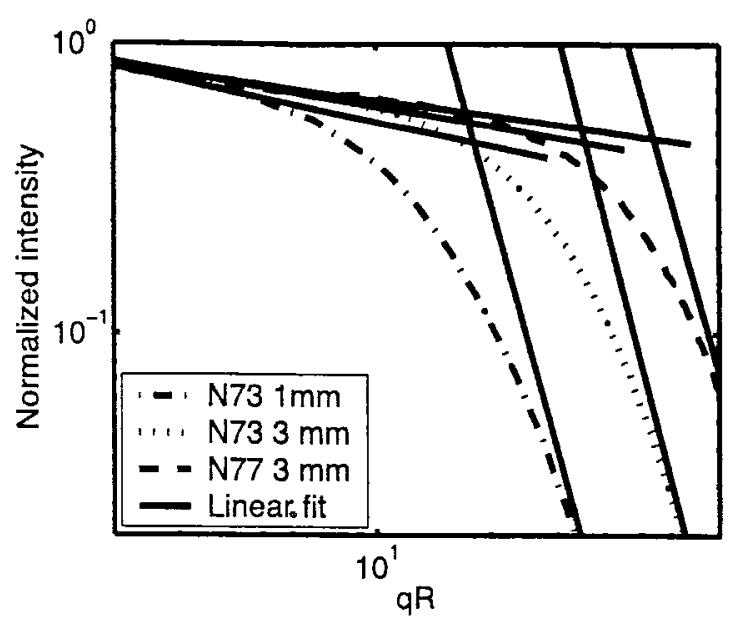

Fig. 9. (a) Comparison of ray-tracing simulation and measured scattering intensities for a normal angle of incidence for all four samples. (b) Crossover regions for three of the simulated scattering intensities versus $q R$. Linear fits for low and high $q R$ regions are shown. Values for the 1-mm N77 sample have been left out because of their similarity to those of the $3-\mathrm{mm}$ N73 sample.

contribution to the term $1-R_{\text {tot }}-T_{\text {tot }}$ is due to side loss. ${ }^{1}$

$$
\bullet
$$

The spectra in Fig. 5(c) show an almost constant level for all angles of incidence except for the highest, $70^{\circ}$. This is an effect of the combination of low TRIMM particle concentration and thin sheet thickness, which results in negligible side loss at low angles of incidence. Figures 5(d), 6( $)$, and 6(d) all show a continuous increase in the side-loss term with increasing angle of incidence, which is expected. A comparison of Fig. 5(d) (thick sheet with a low TRIMM concentration) and Fig. 6(c) (thin sheet with a high TRIMM concentration) indicates that thickness plays a larger role in the side loss than does TRIMM concentration. Comparing the transmittance and reflectance with the angle of incidence for a fixed wavelength, as shown in Fig. 7, makes the importance of sample thickness for side loss even more obvious. This is certainly due to the fact that the incident light hits the sample farther away from the port, giving it a longer distance over which to scatter away from the entrance port.

\section{B. Angle-Resolved Measurements at Oblique Angles of Incidence}

Results for angle-resolved scattering at oblique angles of incidence are presented in Fig. 8. Normal angle-of-incidence results have been included for comparison. These results confirm that the scattering is symmetric at the normal angle of incidence, as expected for an isotropically scattering sample. Asymmetric broadening of the scattering profile is seen as the angle of incidence increases. It is interesting to note the similarity between Figs. 8(b) and $8(\mathrm{c})$, which again indicates that the number of scattering events through a combination of thickness and concentration is what produces the scattering profile.
This outcome is consistent with all other results, but it was of interest to verify that the thickness would not result in peak broadening as a result of a longer path length inside the sample.

\section{Ray-Tracing Simulations for Angle-Resolved Measurements at a Normal Angle of Incidence}

A comparison of measured data and ray-tracing results is shown in Fig. 9(a). The slope toward higher $q R$ is close to the predicted power -4 behavior. The phase factor, $\rho$, is 2.5 and 3.4 for 7.5 - and $10-\mu \mathrm{m}$ radii, respectively, indicating the other two power regimes to be insignificant. A more detailed look at the powerlaw crossover regions is given in Fig. 9(b). The most noteworthy detail is that the power-law crossover point seems to occur at approximately the same relative intensity for all combinations of sheet thickness and TRIMM particle concentration, whereas the scattering profile is shifted almost only as concentration and thickness increase toward higher $q R$ without much distortion of its shape. Another, more obvious, characteristic is that the $3-\mathrm{mm} \mathrm{N73}$ sample and the 1-mm N77 sample are extremely similar, indicating that the number of scattering events is critical for the profile, regardless whether this number comes from a high concentration or an increased thickness.

\section{Conclusions and Outlook}

A thorough characterization of a translucent sheet with TRIMM particles has been carried out for a combination of different concentrations and sheet thicknesses. Hemispheric and angle-resolved optical properties at oblique angles of incidence agree with earlier measurements at normal incidence, which implies that the material is well suited to use in lighting applications.

The possibility of simulating the scattering profile 
of a sheet of given thickness, particle concentration, and particle refractive index is useful for the design of new materials and structures by use of TRIMM particles. The inverse situation in which material parameters can be obtained from a desired scattering profile is still not possible and may not be achievable considering the complexity of the scattering in these materials.

All results, experimental and from simulations, indicate that the combination of sheet thickness and particle concentration that produces the effective number of scattering events is what controls the scatAQ:D tering profile. We are currently investigating ways in which the scattering profile scales with this scattering number, but these investigations have not been completed.

Simulation of the scattering profile for oblique angles of incidence can be handled by the ray-tracing simulation program because both $\theta$ and $\phi$ for each ray are calculated, but the lack of symmetry found for a normal angle of incidence makes it harder to draw any general conclusions.

This study was financed by the Swedish Foundation for Strategic Research through the research school Advanced Micro Engineering, The Swedish Research Council for Environment, Agricultural Sci- ences and Spatial Flanning, and an Australian Research Council Linkage grant.

\section{References}

1. G. B. Smith, J. C. Jonsson, and J. Franklin, "Spectral and global diffuse properties of high-performance translucent polymer sheets for energy efficient lighting and skylights," Appl. Opt. 42, 3981-3991 (2003).

2. K. Grandin and A. Roos, "Evaluation of correction factors for transmittance measurements in single-beam integration spheres," Appl. Opt. 33, 6098-6104 (1994).

3. P. Nostell, A. Roos, and D. Rönnow, "Single-beam integrating sphere for reflectance and transmittance measurements versus angle of incidence in the solar wavelength range on diffuse and specular samples," Rev. Sci. Instrum. 70, 2481-2494 (1999).

4. C. M. Sorensen and D. Fischbach, "Patterns in Mie scattering," Opt. Commun. 173, 145-153 (2000).

5. C. M. Sorensen and D. Shi, "Patterns in the ripple structure of Mie scattering," J. Opt. Soc. Am. A 19, 122-125 (2002).

6. C. Oh and C. M. Sorensen, "Scaling approach for the structure factor of a generalized system of scatterers," J. Nanopart. Res. 1, 369-377 (1999).

7. J. C. Jonsson, A. Roos, and G. B. Smith, "Light trapping in translucent samples and its effect on the hemispherical transmittance obtained by an integrating sphere," in Optical Diagnostic Methods for Inorganic Materials III, L. M. Hanssen, ed., Proc. SPIE 5192, 91-100 (2003).

8. G. B. Smith, D. C. Green, G. McCredit, M. Hossain, P. D. Swift and M. Luther, "Optical characterisation of materials and systems for daylighting," Renew. Energy 22, 85-90 (2001). 